\title{
T cell control in autoimmune bullous skin disorders
}

\author{
Michael Hertl, Rüdiger Eming, and Christian Veldman
}

Department of Dermatology and Allergology, Philipps University, Marburg, Germany.

\begin{abstract}
Autoimmune bullous disorders are a group of severe skin diseases characterized clinically by blisters and erosions of skin and/or mucous membranes. A hallmark of these disorders is the presence of IgG and occasionally IgA autoantibodies that target distinct adhesion structures of the epidermis, dermoepidermal basement membrane, and anchoring fibrils of the dermis. This Review focuses on the potential role of autoreactive $T$ cells in the pathogenesis of these disorders. Pemphigus vulgaris (PV) and bullous pemphigoid (BP) are the best-characterized bullous disorders with regard to pathogenesis and T cell involvement. Activation of autoreactive T cells in PV and BP is restricted by distinct HLA class II alleles that are prevalent in individuals with these disorders. Autoreactive T cells are not only present in patients but can also be detected in healthy individuals. Recently, a subset of autoreactive $T$ cells with remarkable regulatory function was identified in healthy individuals and to a much lesser extent in patients with PV, suggesting that the occurrence of autoimmune bullous disorders may be linked to a dysfunction of Tregs.
\end{abstract}

Autoimmune bullous skin disorders are characterized by the presence of autoantibodies that target distinct adhesion molecules of the epidermis and dermoepidermal basement membrane zone, leading to a loss of adhesive function of the target antigen(s) and, clinically, to the appearance of blisters and erosions $(1,2)$ (Figures 1 and 2). Desmogleins are transmembranous components of desmosomes, adhesion units specialized in conferring epidermal keratinocyte cohesion and linked to intercellular molecules of the desmosomal plaque, which in turn interact with components of the cytoskeleton (Figure 1). In pemphigus, IgG autoantibodies against desmoglein 3 (Dsg3) and Dsg1 lead to loss of desmosomal adhesion of epidermal keratinocytes and intraepidermal blister formation (Figure 2). In the pemphigoids, IgG autoantibodies against components of the dermoepidermal basement membrane such as bullous pemphigoid (BP) antigen 180 (BP180; also referred to as BP antigen 2 and type XVII collagen), BP antigen 230 (BP230; also referred to as BP antigen 1), and laminin 5 interfere with the adhesion of basal epidermal keratinocytes to the dermoepidermal basement membrane zone (Figure 1). BP180 and BP230 are transmembranous and intracellular components, respectively, of hemidesmosomes of basal epidermal keratinocytes, while laminin 5 is a ligand of BP180 located in the lamina lucida of the basement membrane zone (Figure 1). The autoantigen of epidermolysis bullosa, type VII collagen, is the major component of anchoring fibrils that connect the dermoepidermal basement membrane with interstitial collagen bundles of the dermis (Figure 1). The autoantigen of dermatitis herpetiformis, epidermal transglutaminase, is targeted by autoantibodies of the IgA class in the papillary dermis (Figure 2).

Based on the specificity of the targeted antigens, several clinically and immune serologically distinct bullous disorders have been defined (Figure 2). Pemphigus and pemphigoid are considered to

Nonstandard abbreviations used: BP, bullous pemphigoid; BP180, BP antigen 180; BP230, BP antigen 230; Dsg, desmoglein; NC16A, noncollagenous domain 16; PF, pemphigus foliaceus; PG, pemphigoid gestationis; PV, pemphigus vulgaris; Tr1, type 1 Treg.

Conflict of interest: The authors have declared that no conflict of interest exists. Citation for this article: J. Clin. Invest. 116:1159-1166 (2006). doi:10.1172/JCI28547. be prototypic bullous disorders based on their well-characterized immune response-mediated pathogenesis (3-5). Apart from pemphigus and BP, there is only circumstantial evidence that autoreactive $T$ cells are present and involved in the pathogenesis of the autoimmune bullous disorders epidermolysis bullosa acquisita $(6,7)$ and dermatitis herpetiformis $(8,9)$. In pemphigus vulgaris (PV) and BP, autoreactive $\mathrm{CD} 4^{+} \mathrm{T}$ lymphocytes that are presumably crucial in initiating the autoimmune response recognize distinct epitopes of the extracellular portions of Dsg3 and BP180, components of desmosomal and hemidesmosomal adhesion complexes of human skin, respectively. Dsg3- and BP180-reactive $\mathrm{T}$ cells produce Th 2 cytokines such as IL-4, IL-5, and IL-13 and presumably foster the production of autoantibodies of the Th2dependent IgG4 subtype, which are preferentially seen in active stages of these disorders (Figure 3).

\section{Autoreactive T lymphocytes in pemphigus}

$\mathrm{PV}$ is a severe blistering disorder of the mucous membranes and skin associated with IgG autoantibodies against the desmosomal adhesion molecules Dsg3 and Dsg1 (10) (Figures 1 and 2). Autoantibodies against Dsg3 and Dsg1 are critical in the pathogenesis of PV since their transfer into newborn mice induces a phenotype resembling PV $(11,12)$. Peripheral CD4 ${ }^{+}$ $\mathrm{T}$ cell responses, and occasionally $\mathrm{CD}^{+} \mathrm{T}$ cell responses, to the ectodomain of Dsg3 were identified in PV patients by several independent investigators (13-15); however, the phenotype, cytokine profile, immunogenetic restriction, and epitope specificity of these autoreactive $T$ cell responses varied. Dsg3-reactive Th1 $(13)$ and Th2 $(15,16)$ cells were identified that recognized portions of the extracellular domain of Dsg3 in the context of PV-associated HLA class II alleles. By ELISPOT assay, Dsg3-specific autoreactive Th1 and Th2 cells were detected at similar frequencies in acute onset PV (17). By magnetic cell sorting (MACS) cytokine secretion assay, Dsg3-reactive Th2 cells were detected at similar frequencies in acute onset, chronic active, and remittent PV, while the number of autoreactive Th1 cells exceeded that of Th 2 cells in chronic active PV (18). Autoreactive Th1 and Th2 cells may be involved in the regulation of the production of pathogenic autoantibodies by B cells in PV since sera of patients 
A

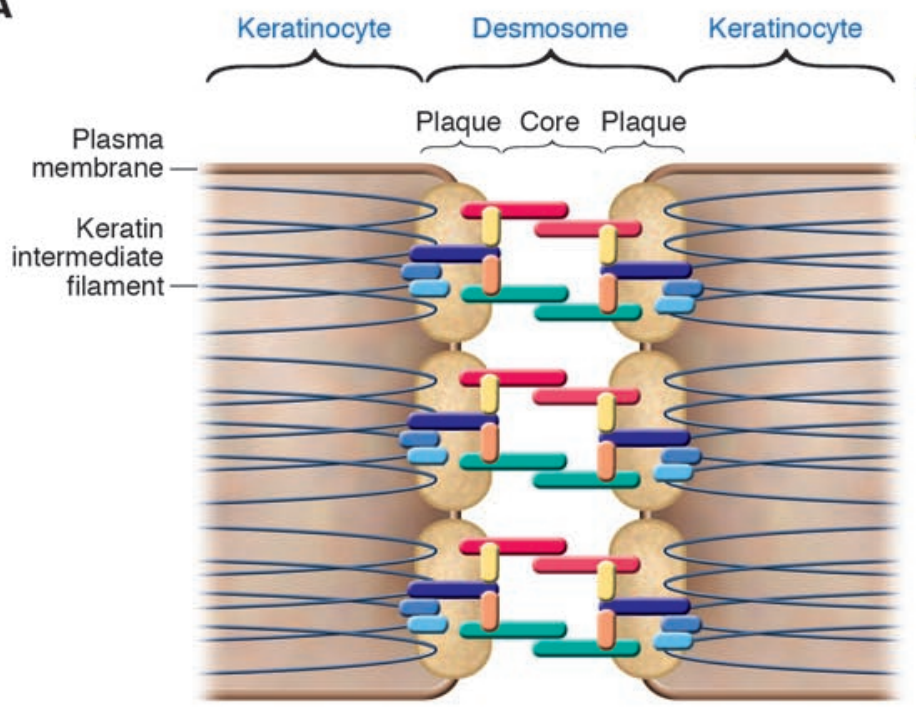

Autoantigens

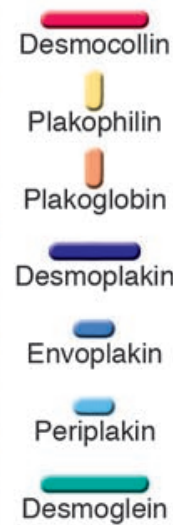

B

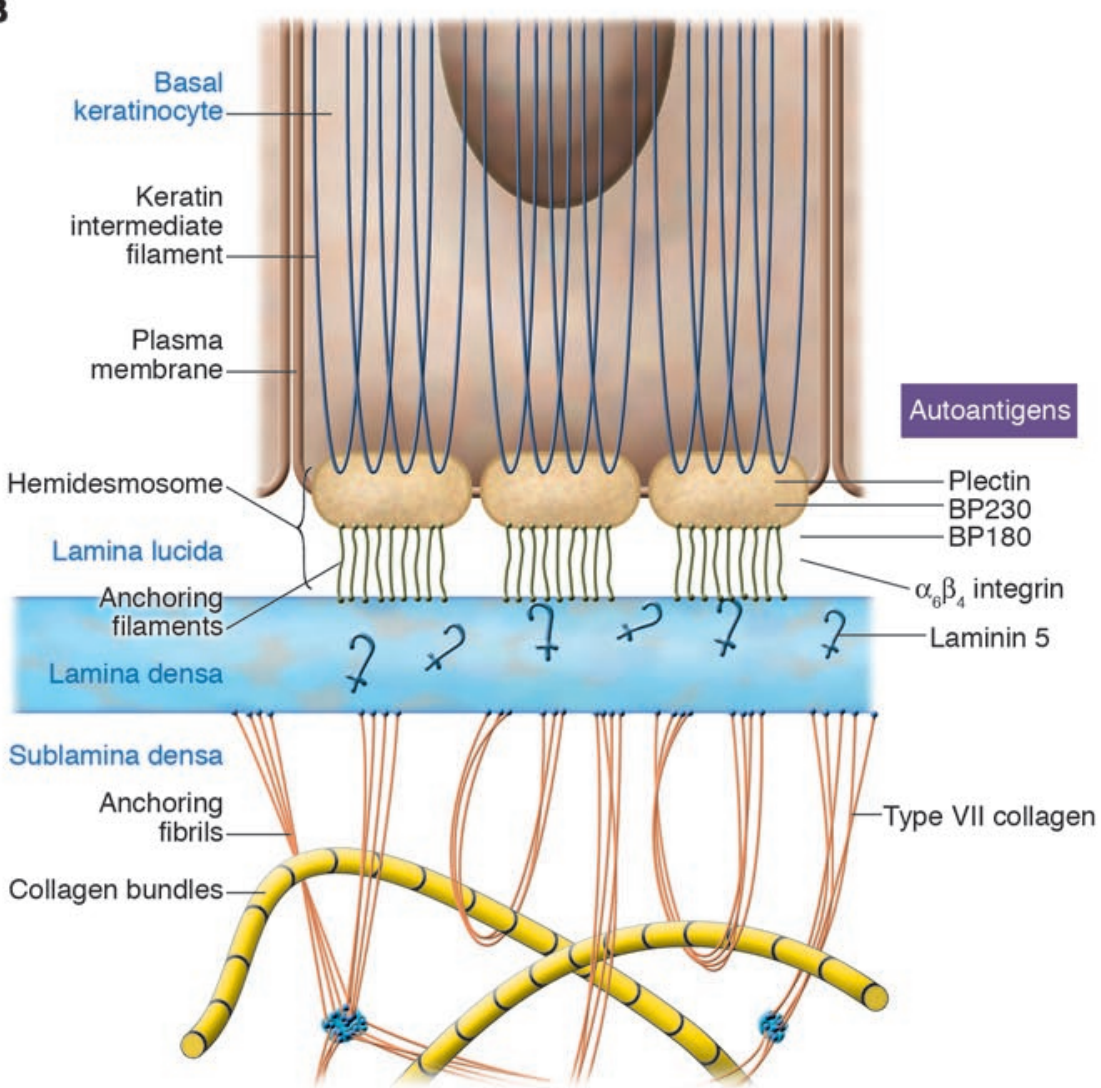

\section{Figure 1}

Autoantigens of human autoimmune bullous skin disorders and their role in epidermal and dermoepidermal adhesion. (A) Desmosomes. The integrity of epidermal cell cohesion is largely dependent on desmosomes, plaquelike intercellular adhesion structures that connect transmembranous adhesion molecules such as the desmogleins and desmocollins with keratins of the cytoskeleton through interaction with intracellular components of the desmosomal plaque such as desmoplakin, plakoglobin, plakophilin, envoplakin, and periplakin. In addition to Dsg3 and Dsg1, which are autoantigens in pemphigus, all the other aforementioned components of desmosomes have been identified as autoantigens of different clinical variants of pemphigus. (B) Hemidesmosomes and components of the dermoepidermal basement membrane. Basal keratinocytes adhere to the basement membrane zone by the interaction of cytoplasmic and transmembranous components of hemidesmosomes, such as BP230 and BP180 and $\alpha_{6} \beta_{4}$ integrin, with ligands such as laminin 5 located in the lamina lucida and lamina densa of the dermoepidermal basement membrane zone. The intracellular hemidesmosomal components BP230 and plectin are linked to keratins of the cytoskeleton and interact with the cytoplasmic domains of BP180 and $\alpha_{6} \beta_{4}$ integrin, which in turn interact with laminin 5 via their ectodomains. Type VII collagen is the major component of anchoring fibrils, which link the basement membrane to interstitial dermal collagen fibers by direct interaction with laminin 5 in the lamina densa of the basement membrane. Figure modified from ref. 58. with PV contain Th1-regulated IgG1 and Th2-regulated IgG4 autoantibodies directed against Dsg3 (Figure 3) $(19,20)$.

PV is associated with HLA-DR $\beta 1 * 0402$ and HLA-DQ $\beta 1 * 0503$, while the endemic variant of pemphigus foliaceus (PF), fogo selvagem, which is found in limited areas of South America, is characterized by a prevalence of the 2 HLA class II alleles, DR $\beta 1 * 0402$ and DR $\beta 1 * 0101(21-23)$. We and others found in patients with PV that Th1 and Th2 cell recognition of Dsg3 peptides was restricted by HLA-DR $\beta 1^{*} 0402$ and/or HLA-DQ $\beta 1{ }^{*} 0503$ and that the pro- liferative response of autoreactive Th cells was blocked by anti-DR and anti-DQ antibodies, respectively $(15,18,24,25)$. Occasionally, Dsg3-reactive Th cell clones were also restricted by non-PV-associated HLA class II alleles; however, these alleles were homologous to DR $\beta 1 * 0402$ with regard to peptide-binding motifs (25).

In several studies, we were able to confirm the hypothesis that distinct HLA class II alleles shape the autoimmune response to Dsg3 $(13,24,26)$. Dsg3-reactive Th1 and Th2 clones from PV patients and HLA-matched healthy donors recognized a limited set of Dsg3 

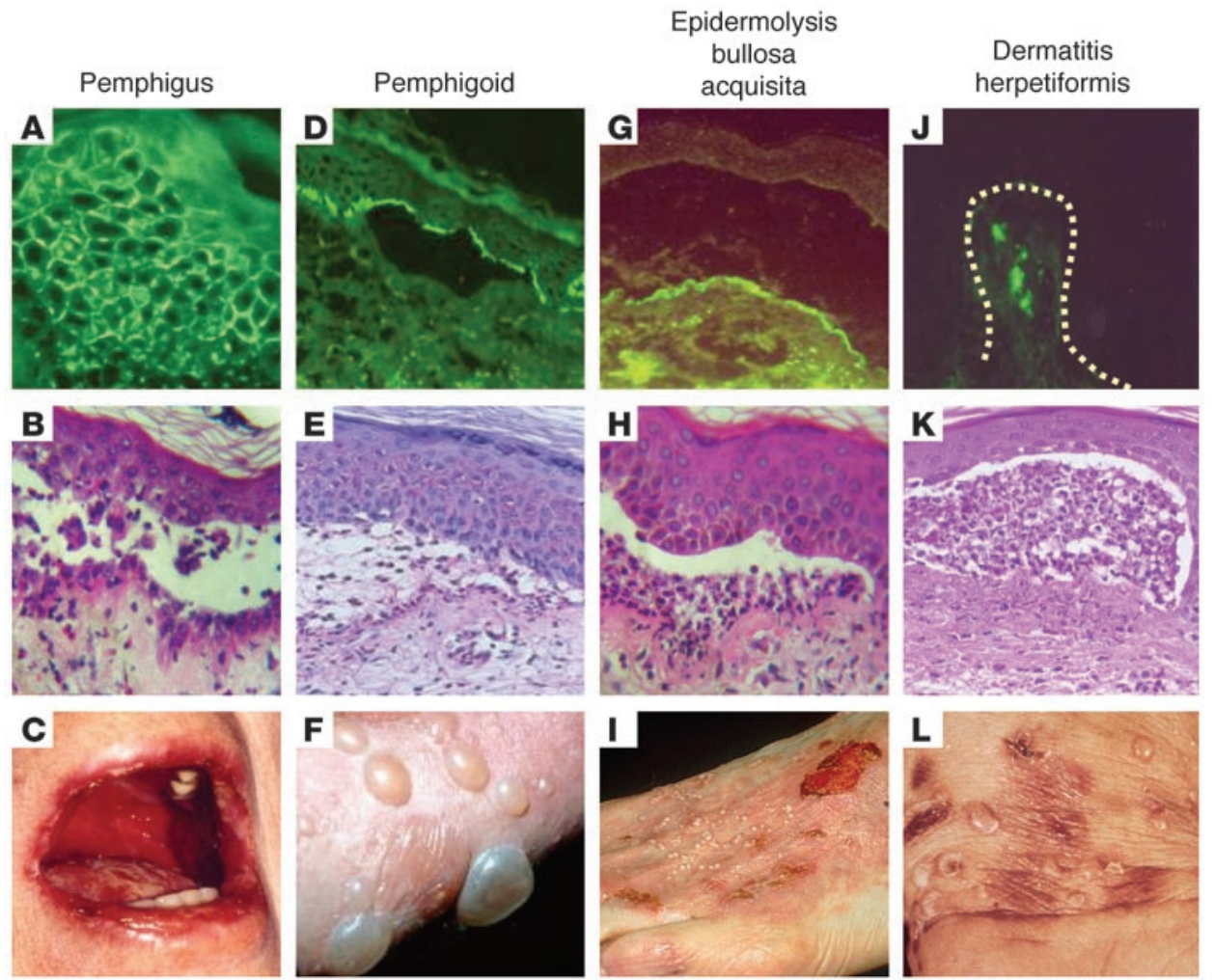

Figure 2

Immunological and clinical characteristics of autoimmune bullous skin disorders. (A-C) Pemphigus is characterized by the presence of IgG (and occasionally IgA) specific for desmosomal target antigens (visualized as an intercellular staining pattern by direct immunofluorescence; $\mathbf{A}$ ) resulting in a loss of intraepidermal adhesion (as shown by histopathology; B) and blisters and/or erosions of the mucous membranes and skin (C). (D-F) In the pemphigoids, including linear IgA bullous dermatosis, IgG (or $\lg A$ ) autoantibodies bind to antigens of hemidesmosomes and the lamina lucida of the dermoepidermal junction $(\mathbf{D})$, resulting in a loss of subepidermal adhesion $(\mathbf{E})$ and tense blisters $(\mathbf{F})$. (G-I) Epidermolysis bullosa acquisita is associated with $\lg$ (and sometimes $\lg A$ ) binding to the anchoring fibrils underneath the lamina densa of the dermoepidermal junction $(\mathbf{G})$, resulting in a subepidermal loss of adhesion $(\mathbf{H})$ and tense blisters with a tendency toward scarring and milia formation (I). (J-L) Dermatitis herpetiformis is associated with deposits in the papillary dermis (dotted line indicates the dermoepidermal junction) of IgA reactive with epidermal transglutaminase $(\mathbf{J})$, a subepidermal loss of adhesion $(\mathbf{K})$, and herpetiform blisters or pruritic papules (L). Magnification, $\times 100$ (A, B, D, E, G, H, J, and K). ous study (28). By ELISPOT assay, IgG-secreting B cells specific for Dsg3 were detected upon in vitro stimulation of peripheral lymphocytes from PV patients with Dsg3. Depletion of the $\mathrm{CD}^{+} \mathrm{T}$ cell subset led to inactivation of IgG-secreting autoreactive B cells. IgG production by the autoreactive $\mathrm{B}$ cells was also abolished when anti-HLA-DR or anti-HLA-DQ monoclonal antibody was added to the cultures. These findings strongly suggest that circulating Dsg3-specific B cells are regulated by HLA class II-restricted autoreactive $\mathrm{CD}^{+} \mathrm{T}$ cells (Figure 3).

Autoreactive $\mathrm{T}$ cells were also identified in the endemic variant of PF, fogo selvagem (29). Peripheral CD4 ${ }^{+} \mathrm{T}$ cells from patients with endemic PF developed a proliferative response to the extracellular portion of Dsg1, the autoantigen of PF, and secreted Th2 cytokines. Using MACS cytokine secretion assay, we identified Dsg1-responsive Th 1 and Th 2 cells at similar frequencies in patients with PF and occasionally also in healthy individuals (30). These findings are in line with the aforementioned observations in PV, indicating that the presence of autoreactive Th cells is not restricted to patients (18). Other clinical pemphigus variants such as IgA pemphigus and paraneoplastic pemphigus have not been thoroughly characterized regarding the presence of autoreactive $\mathrm{T}$ cells, despite the identification of their autoantigens peptides located within the Dsg3 ectodomain, namely DG3 $3_{78-94}$, DG3 $3_{96-112}$, DG3 ${ }_{189-205}, \mathrm{DG}_{205-221}$, and DG3 $250-266$, which were recognized in association with HLA-DR $\beta 1{ }^{*} 0402$ and HLA-DQ $\beta 1{ }^{*} 0503$ (26). All the identified Dsg3 peptides carried a positive charge at position 4 ( $\mathrm{p} 4$ ), which is critical for binding to the negatively charged $\mathrm{p} 4$ pockets of DR $\beta 1 * 0402$ (aa residues at positions DR $\beta 70$ and DR $\beta 71$ ) and DQ $\beta 1 * 0503$ (aa position DQ $\beta 57$; Figure 4) (14). T cells from healthy carriers of the PV-associated HLA class II molecules exhibited $\mathrm{CD}^{+} \mathrm{T}$ cell responses against identical epitopes of the Dsg3 ectodomain (26). This later finding supports the concept that PV is the consequence of a loss of tolerance on the $\mathrm{B}$ cell level rather than on the T cell level (26). TCR analysis revealed that Dsg3-reactive T cells were of oligoclonal origin and expressed a limited set of different TCRs (27), while an independent study found that autoreactive Th clones specific for the putative immunodominant epitope DG3 $3_{96-112}$ carried a greater spectrum of different TCRs (26).

Circumstantial evidence for a critical role of autoreactive $\mathrm{T}$ cells in fostering antibody production was provided by a previ-
(Table 1). There is some evidence that paraneoplastic pemphigus is at least partly mediated by a $\mathrm{CD}^{+} \mathrm{T}$ cell infiltrate in affected epithelia since necrosis of epithelial cells next to a $\mathrm{T}$ cell infiltrate is a hallmark of paraneoplastic pemphigus (31).

\section{Autoreactive T lymphocytes in the pemphigoids}

The pemphigoids are a group of distinct autoimmune subepidermal blistering diseases of the skin associated with IgG antibodies against BP180 and BP230, 2 components of junctional adhesion complexes called hemidesmosomes that are critically involved in the maintenance of dermoepidermal adhesion (Figure 1) (3-5). $\mathrm{BP} 180$ is considered to be the major autoantigen of BP and IgG antibodies against the noncollagenous domain 16 (NC16A) of the BP180 ectodomain, which are detected in more than $90 \%$ of BP patients and have been shown to be pathogenic in vitro and in vivo $(32,33)$. Th 2 and Th1 responses against the BP180 ectodomain were identified in the majority of the studied BP patients in 2 independent studies $(34,35)$. 


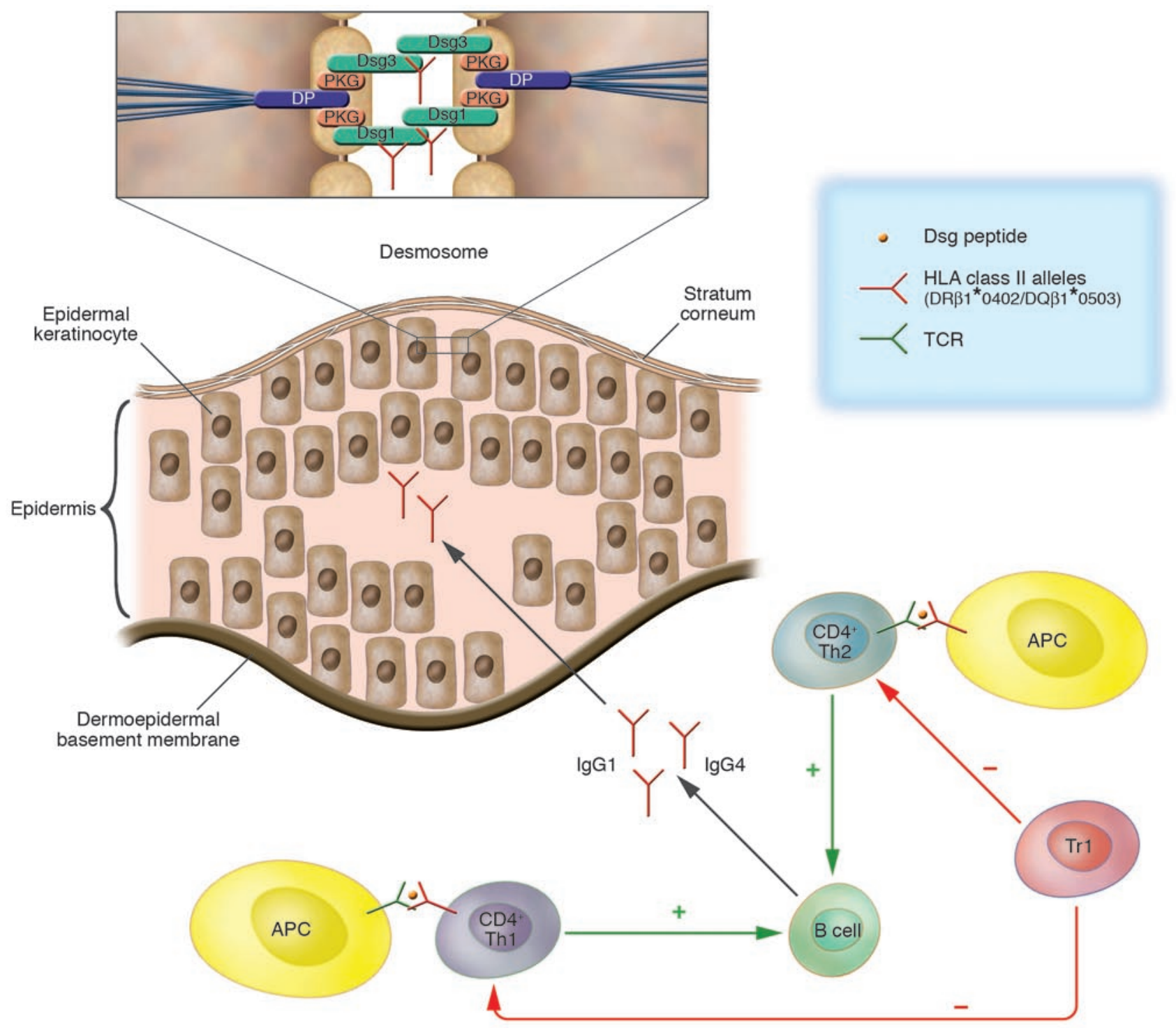

Figure 3

T cell involvement in the immune pathogenesis of PV. PV is the prototype of an autoantibody-mediated immunobullous skin disorder and is characterized by a loss of intraepidermal adhesion primarily caused by autoantibodies belonging to the IgG4 and, to a lesser extent, IgG1 subclasses specific for Dsg3 and Dsg1, components of the desmosomal adhesion complex of epidermal keratinocytes that are connected to the keratin cytoskeleton through interaction with the intracellular plaque proteins plakoglobin (PKG) and desmoplakin (DP; inset). IgG production by autoreactive B cells is presumably regulated by Dsg3- and Dsg1-reactive Th1 and Th2 cells. The Dsg3-reactive Th cells recognize epitopes of the Dsg3 ectodomain in association with the HLA class II alleles HLA-DR $\beta 1^{*} 0402$ and HLA-DQ $\beta 1^{*} 0503$ presented by APCs including dendritic cells and B cells. Dsg3-reactive Th cells that recognize identical epitopes are also found in healthy carriers of the aforementioned PV-associated HLA class II alleles. Thus Tregs may be critical in maintaining peripheral B cell tolerance to Dsg3. This contention is supported by the finding that Dsg3-reactive Tr1s are more frequently detected in healthy individuals than in patients with PV.

Recent studies showed that BP180-reactive Th cells and IgG autoantibodies recognized similar or identical epitopes clustered in distinct regions of the BP180 ectodomain and BP230 (36-38). Specifically, the majority of autoreactive Th2 and Th1 cells and $\mathrm{B}$ cells recognized epitopes within the $\mathrm{NH}_{2}$-terminal portion, followed by reactivity against the $\mathrm{COOH}$-terminal and central portions, of the BP180 ectodomain (37). Of note, $\mathrm{T}$ and $\mathrm{B}$ cell reactivity against the $\mathrm{NH}_{2}$-terminal portion of the BP180 ectodomain was associated with severe BP, with widespread blisters and erosions, while the central portion was more frequently recognized in lim- ited BP, with few blisters and erosions (37). In contrast, less than $50 \%$ of the studied BP patients showed a combined $\mathrm{T}$ and $\mathrm{B}$ cell response against the $\mathrm{COOH}$ - and $\mathrm{NH}_{2}$-terminal globular domains of BP230 (38). This finding is of particular interest in light of the current discussion as to whether the transmembranous adhesion molecule BP180 or the intracellular hemidesmosomal component BP230 is the major autoantigen of BP (Figure 1) (38).

A considerable number of patients with $\mathrm{BP}$ and a clinical variant, pemphigoid gestationis (PG), displayed NC16A-specific peripheral $\mathrm{T}$ cell responses, which were mainly of the Th2 type and a mixed 


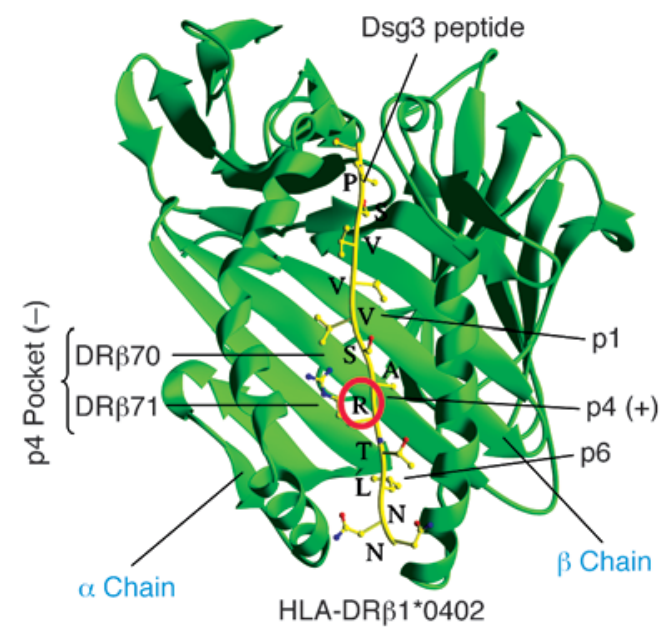

Th1/Th2 type, respectively $(35,39)$. T cell recognition of BP180NC16A seems to be rather heterogeneous since BP180-NC16Aspecific $\mathrm{T}$ cell clones from the BP patients preferentially expressed a TCR different from the TCR expressed by $\mathrm{T}$ cell clones derived from a PG patient (40). Recently, IFN- $\gamma$ secretion by NC16A-specific autoreactive $\mathrm{T}$ cells was also detected in a subset of patients with mucous membrane pemphigoid, suggesting that this clinical variant of $\mathrm{BP}$ is also associated with autoreactive $\mathrm{T}$ cell responses to BP180 (41). Finally, a mixed Th1/Th2 response to BP180 was identified in patients with linear IgA bullous dermatosis. This disorder is clinically distinct from BP and is primarily associated with IgA autoantibodies against a proteolytically cleaved antigen located within the BP180 ectodomain (Figure 1) (42).

A common HLA class II allele, HLA-DQ $\beta 1 * 0301$, seems to be associated with distinct clinical pemphigoid variants (43). Another study found that the association of BP with DQ $\beta 1 * 0301$ was restricted to men (44). In our own experience, several BP180-specific Th1 and Th2 cell clones derived from BP patients were found to be restricted by HLA-DQ $\beta 1 * 0301$ (45). A subset of healthy individ-

\section{Figure 4}

Algorithm for HLA class II-Dsg3 peptide interaction in PV. Shown is the physical interaction of $\mathrm{DR} \beta 1^{*} 0402$ with a representative Dsg3 peptide. HLA-DR $\beta 1^{*} 0402$ differs from other HLA-DR4 molecules by the presence of a negative charge at amino acid residue 71 (DR $\beta 71)$ of the $\beta$ chain, which is a critical binding motif for $T$ cell peptides. Several $T$ cell epitopes of Dsg3, the major autoantigen of PV, have been identified that carry a positively charged amino acid (mostly lysine, $\mathrm{K}$, or arginine, $\mathrm{R}$ ) at relative position 4 ( 4 ; red circle), which serves as an anchor motif to the negatively charged p4 pocket formed by residues DR $\beta 70$ and DR $\beta 71$ of DR $\beta 1^{*} 0402$. Thus DR $\beta 1^{*} 0402$ shapes the fine specificity of the T cell autoimmune response against a limited set of Dsg3 epitopes that fulfill the binding criteria of this specific HLA class II molecule.

uals who carried the BP-associated HLA class II allele DQ $\beta 1{ }^{*} 0301$ also showed BP180-specific T cell responses that were predominantly of the Th1 type (45). Distinct peptide-binding motifs of HLA-DQ $\beta 1 * 0301$ have not yet been defined, nor were the anchor motifs of T cell epitopes of BP180 characterized in detail (38). The presence of IgG autoantibodies against BP180 in the clinical BP variant mucous membrane pemphigoid seems to be associated with the presence of HLA-DQ $\beta 1{ }^{*} 0301$ (46).

\section{In vivo evidence for pathogenic relevance of autoreactive T cells in pemphigus and pemphigoid}

Results obtained from a knockout mouse with a targeted disruption of the $D s g 3$ gene ( $D s g 3^{-/-}$mice) support the concept that the absence or loss of the adhesive function of Dsg3 leads to fragility of the skin, oral erosions, and blisters accompanied by weight loss due to inhibited food uptake and a runted phenotype around day 18 of age (47). Amagai et al. used $D s g 3^{-/-}$mice to establish an active in vivo model of PV by immunizing the $D s g 3^{-/-}$mice with recombinant mouse Dsg3, which finally led to the production of antiDsg3 antibodies (48). Splenocytes from these Dsg3-immunized mice were then transferred into immunodeficient Rag2 ${ }^{-/-}$mice that expressed Dsg3, resulting in the stable production of antiDsg3 IgG and the development of a phenotype resembling PV

Table 1

Autoreactive T and B cell responses in human autoimmune bullous skin disorders

\begin{tabular}{|c|c|c|c|c|}
\hline Disorder & Autoantibodies & $\begin{array}{l}\text { Autoantigens recognized } \\
\text { by autoreactive } T \text { cells }\end{array}$ & T cell subset & Reference \\
\hline \multirow[t]{6}{*}{ PV } & $\begin{array}{l}\lg G 4 \text {, IgG1, occasionally } \lg A \\
\text { (in IgA pemphigus) }\end{array}$ & Dsg3 peptides & PBMC & (14) \\
\hline & & Dsg3 & Th1, Th2 & $(15,16,18)$ \\
\hline & & Dsg3, Dsg1 & Th1, Th2 & $(13,25)$ \\
\hline & & Dsg3, Dsg3 peptides & Th1, Th2 & (26) \\
\hline & & Dsg3 & Th1, Th2, Tr1 & $(54,56)$ \\
\hline & & Dsg3 & CD8+CD28- Treg & $(55)$ \\
\hline \multirow{3}{*}{ BP } & $\lg \mathrm{G} 4, \lg \mathrm{G} 1$ & Dsg1 & Th1, Th2 & $(29,30)$ \\
\hline & IgG1, IgG4, occasionally IgA & BP180 & Th1, Th2 & $(34,35)$ \\
\hline & & BP180, BP230 & Th1, Th2 & $(38)$ \\
\hline \multirow[t]{2}{*}{$P G$} & $\lg \mathrm{G} 1, \lg \mathrm{G} 3$ & BP180 & Th1, Th2 & (39) \\
\hline & & BP230 & ND & \\
\hline \multirow[t]{2}{*}{ Mucous membrane pemphigoid } & $\lg \mathrm{G} 1, \lg \mathrm{G} 4$, occasionally $\lg A$ & BP180 & Th1 & $(41)$ \\
\hline & & Laminin $5, \beta_{4}$ integrin, BP230 & ND & \\
\hline \multirow[t]{2}{*}{ Linear IgA bullous dermatosis } & $\lg A$ & BP180 & Th1, Th2 & (42) \\
\hline & & BP230 & ND & \\
\hline
\end{tabular}

ND, not determined. 


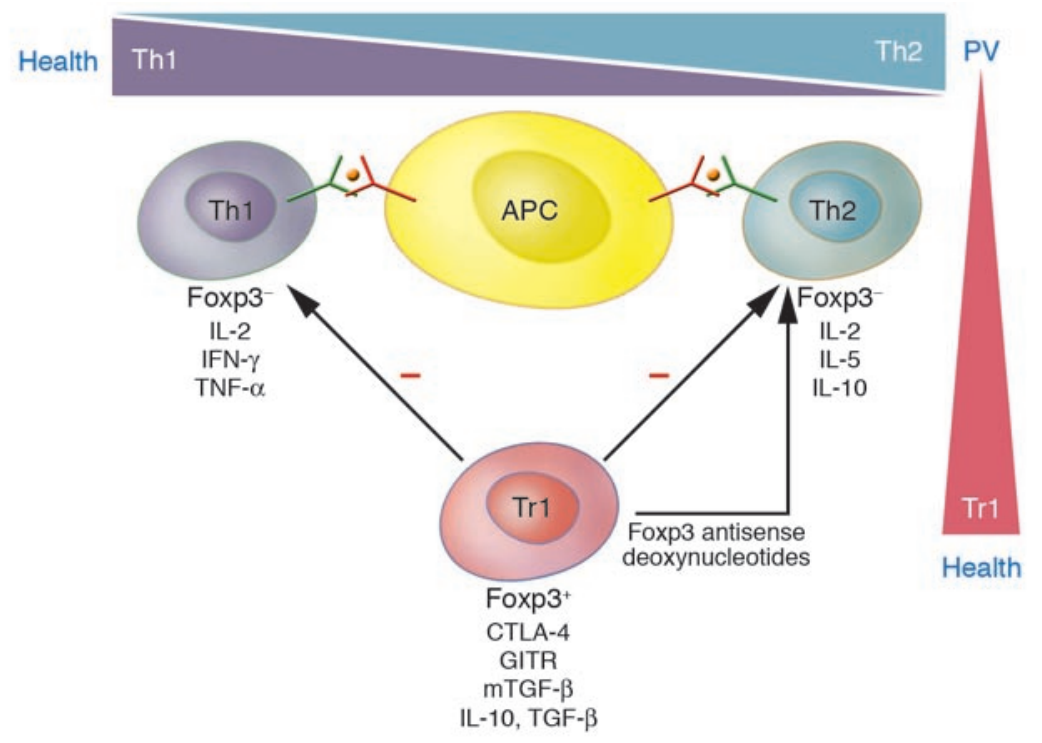

Figure 5

Putative T cell network in PV and health. Dsg3- and Dsg1-reactive Th1 and Th2 cells are present in patients with PV and healthy carriers of PV-associated HLA class II alleles, which recognize identical epitopes of the Dsg3 ectodomain presented by APCs such as dendritic cells and B cells. There is a predominance of Dsg3-reactive Th2 cells in PV and of autoreactive Th1 cells in healthy individuals. In addition, Tr1s specific for Dsg3 are present at higher frequencies in healthy individuals than in PV patients. These Tr1s express the phenotypic markers glucocorticoid-induced TNF receptor (GITR), membrane-bound TGF- $\beta$ (mTGF- $\beta$ ), and cytotoxic T cell antigen-4 (CTLA-4) and inhibit the activation of Dsg3-specific autoreactive Th1 and Th2 cells via the secretion of IL-10 and TGF- $\beta$ and the uptake of exogenous IL-2 produced by the Th cells. Inactivation of the regulatory transcription factor Foxp3 by antisense deoxynucleotides converts Tr1s into a Th2-like population with regard to cytokine profile, loss of inhibitory function on Th cells, and the ability to develop a proliferative response to Dsg3. Thus an imbalance of the putative relationship between autoreactive Th and Tr1 cells may be critical in the pathogenesis of PV. Hence Dsg-specific Tr1s may be critical for the maintenance of tolerance against Dsg3 and Dsg1 in health and the restoration of tolerance against these autoantigens in remittent PV.

immunization of the DR0402-DQ8-transgenic mice with human Dsg3 recognize epitopes of Dsg3 that exhibit HLA class II anchor motifs identical to the Dsg3 epitopes previously identified in humans $(26,52)$. Thus the present mouse model will hopefully facilitate the study of the regulation of HLA class II-restricted T cell recognition of Dsg3 and T cell help for the production of pathogenic antibodies in vivo.

\section{Potential regulatory network of autoaggressive T cells in pemphigus}

There is now compelling evidence that $\mathrm{CD}^{+} \mathrm{T}$ cells specialized in suppressing immune responses play a critical role in immune regulation (53). Tregs exert a dominant effect in controlling autoimmunity and maintaining peripheral tolerance. The best described of these cells are the naturally occurring $\mathrm{CD} 4{ }^{+} \mathrm{CD} 25^{+}$ Tregs that inhibit Th activation in a cell contact-dependent and non-Ag-specific manner; this $\mathrm{T}$ cell subset represents up to $10 \%$ of peripheral $\mathrm{CD}^{+} \mathrm{T}$ cells (53). In addition to the naturally occurring $\mathrm{CD} 4{ }^{+} \mathrm{CD} 25^{+}$Tregs, which have been shown to be continuously produced within the thymus, other $\mathrm{T}$ cell subsets bearing suppressive capacity have been described. Among those the most prominent are type 1 Tregs $(\operatorname{Tr} 1 \mathrm{~s})$, which have been shown to be induced upon antigen exposure under certain tolerogenic conditions and are characterized by the produc-

including oral erosions with suprabasilar acantholysis. Using this mouse model of PV, Tsunoda et al. (49) demonstrated that pathogenic anti-Dsg3 IgG production in the Rag2-/- recipients was only observed when both T and B cells were transferred from the Dsg3immunized $\mathrm{Dsg}^{-/-}$mice. Thus loss of tolerance against Dsg3 in this in vivo model of $\mathrm{PV}$ required the presence of both autoreactive $\mathrm{T}$ and $\mathrm{B}$ cells. Furthermore, these findings illustrate the importance of a direct interaction of Dsg3-reactive CD4 ${ }^{+} \mathrm{T}$ cells and $\mathrm{B}$ cells for Dsg3-specific antibody production. Aoki-Ota et al. went on to show that the adoptive transfer of $\mathrm{T}$ and $\mathrm{B}$ splenocytes from nonimmunized $\mathrm{Dsg}^{-/-}$mice (which recognized Dsg3 as a foreign antigen) also induced the stable production of anti-Dsg3 IgG and a PV-like phenotype in the recipient $D s g 3^{+/+} R a g 2^{-/-}$mice (50).

Our group explored an HLA class II transgenic mouse model to characterize $\mathrm{CD}^{+} \mathrm{T}$ cell recognition of human Dsg3 epitopes in vivo (51). These mice express the PV-associated HLA class II haplotype, $H L A-D R A * 0101$ and $D R B 1 * 0402$ and $H L A-D Q A^{*} 0301$ and $D Q B 1 * 0302$ (DQ8), which are both regulated by the I-E $\alpha$ promoter. These mice also carry the human CD4 receptor and are deficient in the major murine MHC class II molecules (I-A $\left.\beta^{-/-}\right)(52)$. Immunizing these HLA-DR0402-DQ8-transgenic mice with recombinant human Dsg3 protein led to the induction of both Dsg3-specific $\mathrm{CD}^{+} \mathrm{T}$ cell responses and Dsg3-reactive antibody production (51). $\mathrm{CD}^{+} \mathrm{T}$ cell hybridomas derived from draining lymph nodes after tion of the immunosuppressive cytokines IL-10 and TGF- $\beta$ (53). Recent progress has been made in the characterization of $\operatorname{Tr} 1 \mathrm{~s}$ in terms of isolation and induction.

At present, a potential imbalance of $\mathrm{CD} 4^{+} \mathrm{CD} 25^{+}$Tregs has not been extensively investigated in pemphigus. In a recent study, a subset of Dsg3-reactive, IL-10-secreting T cells was identified that was present in the majority of healthy carriers of PV-associated HLA class II alleles but in less than $20 \%$ of the studied PV patients (Figure 5) (54). This distinct T cell subset fulfilled the criteria of $\operatorname{Tr} 1 \mathrm{~s}$ and suppressed the proliferative response of Dsg3-specific autoreactive Th cells by secretion of IL-10 and TGF- $\beta$ in a cell contact-independent manner (54). Sinha and coworkers identified a subset of $\mathrm{CD} 8^{+} \mathrm{CD} 28^{-} \mathrm{T}$ cells with potential Treg function that were present in newly remittent $\mathrm{PV}$ patients but not in active or fully remittent PV patients (55). These findings suggest that different subsets of Tregs may be involved in the maintenance of peripheral tolerance to Dsg3 in healthy individuals and in the restoration of tolerance against Dsg3 in patients with remittent PV (Figure 5).

Little is known about the transcriptional regulation of $\operatorname{Tr} 1 \mathrm{~s}$ in autoimmune disorders in general. Recently Foxp3, which encodes a transcription repressor, has been shown to be expressed by Tregs and to be critical for Treg development. Our group showed that expression of Foxp3 mRNA and protein is an inherent fea- 
ture of human Tr1s specific for Dsg3 (56). These Dsg3-specific $\operatorname{Tr} 1 \mathrm{~s}$ expressed phenotypic markers of Tregs such as cytotoxic $\mathrm{T}$ cell antigen-4, glucocorticoid-induced TNF receptor, and membrane-bound TGF- $\beta$ and inhibited the activation of autoreactive Th1 and Th2 cells via the secretion of IL-10 and TGF- $\beta$ (Figure 5). Antisense-driven inactivation of Foxp 3 mRNA induced a shift of Dsg3-specific $\operatorname{Tr} 1$ clones toward a Th2-like phenotype (Figure 5): Foxp3 antisense oligodeoxynucleotide-treated Dsg3-specific $\operatorname{Tr} 1$ cell clones secreted IL-2 and the Th2 cytokines IL-5 and IL-10, but no IFN- $\gamma$ or TNF- $\alpha$ (56). Moreover, they lost their phenotypic Treg markers and inhibitory effect on Th1 and Th2 cells and showed a vigorous proliferative response to Dsg3. This finding suggests that there is a potential relationship between autoreactive Th2 cells and $\mathrm{Tr} 1 \mathrm{~s}$ in PV, which may be derived from a common precursor cell. There is also evidence that Th cells may be converted into Tr1s by bystander mechanisms involving cell-cell contact with naturally occurring $\mathrm{CD} 4^{+} \mathrm{CD} 25^{+}$Tregs (57).

In vivo, there may be a physiological balance between Dsg3responsive autoreactive Th2 cells and Dsg3-specific $\operatorname{Tr} 1 \mathrm{~s}$ required for the maintenance of peripheral tolerance, since a high ratio of IL- $10^{+} \mathrm{Th} 2 / \operatorname{Tr} 1$ cells was seen in PV patients, while a low IL- $10^{+} \mathrm{Th} 2 / \operatorname{Tr} 1$ ratio was commonly found in Dsg3responsive healthy carriers of PV-associated HLA class II alleles (Figure 5) (54). These findings in PV provide a sound explanation as to why B cell tolerance against Dsg3 exists in healthy individuals who carry autoreactive $T$ cells with epitope specificity identical to that of patients.

1. Beutner, E.H., Jordon, R.E., and Chorzelski, T.P. 1968. The immunopathology of pemphigus and bullous pemphigoid. J. Invest. Dermatol. 51:63-80.

2. Yancey, K.B., and Egan, C.A. 2000. Pemphigoid: clinical, histologic, immunopathologic, and therapeutic considerations. JAMA. 284:350-356.

3. Stanley, J.R. 1989. Pemphigus and pemphigoid as paradigms of organ-specific, autoantibody-mediated diseases. J. Clin. Invest. 83:1443-1448.

4. Hertl, M. 2000. Humoral and cellular autoimmunity in autoimmune bullous skin disorders. Int. Arch. Allergy Immunol. 122:91-100.

5. Borradori, L., and Sonnenberg, A. 1996. Hemidesmosomes: roles in adhesion, signaling and human diseases. Curr. Opin. Cell Biol. 8:647-656.

6. Egan, C.A., Brown, M., White, J.D., and Yancey, K.B. 2001. Treatment of epidermolysis bullosa acquisita with the humanized anti-Tac mAb daclizumab. Clin. Immunol. 101:146-151.

7. Hallel-Halevy, D., Nadelman, C., Chen, M., and Woodley, D.T. 2001. Epidermolysis bullosa acquisita: update and review. Clin. Dermatol.19:712-718.

8. Sardy, M., Karpati, S., Merkl, B., Paulsson, M., and Smyth, N. 2002. Epidermal transglutaminase (TGase 3 ) is the autoantigen of dermatitis herpetiformis. J. Exp. Med. 195:747-757.

9. Caproni, M., Cardinali, C., D’Agata, A., Selvaggi, W., and Fabbri, P. 2002. Serum eosinophil cationic protein, myeloperoxidase, tryptase, eotaxin and Th2-like cytokines in Dermatitis herpetiformis. Int. Arch. Allergy Immunol. 128:67-72.

10. Hertl, M., and Veldman, C. 2001. Pemphigus - paradigm of autoantibody-mediated autoimmunity. Skin Pharmacol. Appl. Skin Physiol. 14:408-418.

11. Amagai, M., Karpati, S., Prussick, R., Klaus-Kovtun, V., and Stanley, J.R. 1992. Autoantibodies against the amino-terminal cadherin-like binding domain of pemphigus vulgaris antigen are pathogenic. J. Clin. Invest. 90:919-926.

12. Anhalt, G.J., Labib, R.S., Voorhees, J.J., Beals, T.F., and Diaz, L.A. 1982. Induction of pemphigus in neonatal mice by passive transfer of IgG

\section{Conclusions}

There is increasing evidence for a critical role of autoreactive T cells in the regulation of the production of pathogenic autoantibodies in 2 prototypes of autoimmune bullous disorders - pemphigus and pemphigoid. Both disorders are presumably associated with a dysregulation of Th1 and Th2 responses against cutaneous autoantigens, since Th2 responses are primarily associated with disease and Th1 responses are found in healthy individuals. In addition, PV may be the consequence of an imbalance between Dsg3-responsive Th2 cells and Tregs specialized in counter-regulating the devastating $\mathrm{T}$ cell autoimmune response. Thus autoreactive Tregs may represent an ideal tool to specifically restore immune tolerance in autoimmune bullous skin disorders.

\section{Acknowledgments}

We wish to thank Stefan Hörster for providing histological images and Andrea Niedermeier for critically reading the manuscript. This study was supported by grants from the Deutsche Forschungsgemeinschaft (He 1602/6-2 to M. Hertl; He 1602/7-1 and He 1602/72 to C. Veldman and M. Hertl; and RM80/1-1 to R. Eming), the Wilhelm Sander-Stiftung (Nr. 2004.120.1 to M. Hertl), and the Deutsche Dermatologische Gesellschaft (to R. Eming).

Address correspondence to: Michael Hertl, Department of Dermatology and Allergology, Philipps University, Deutschhausstrasse 9, D-35033 Marburg, Germany. Phone: 49-6421-28-66280; Fax: 496421-28-62902; E-mail: hertl@med.uni-marburg.de. from patients with the disease. N. Engl. J. Med. 306:1189-1196.

13. Hertl, M., et al. 1998. Recognition of desmoglein 3 by autoreactive $T$ cells in pemphigus vulgaris patients and normals. J. Invest. Dermatol. 110:62-66.

14. Wucherpfennig, K.W., et al. 1995. Structural basis for major histocompatibility complex (MHC)linked susceptibility to autoimmunity: charged residues of a single MHC binding pocket confer selective presentation of self-peptides in pemphigus vulgaris. Proc. Natl. Acad. Sci. U. S. A. 92:11935-11939.

15. Lin, M.S., et al. 1997. Development and characterization of desmoglein-3 specific T cells from patients with pemphigus vulgaris. J. Clin. Invest. 99:31-40.

16. Rizzo, C., et al. 2005. Direct characterization of human $T$ cells in pemphigus vulgaris reveals elevated autoantigen-specific Th2 activity in association with active disease. Clin. Exp. Dermatol. 30:535-540.

17. Eming, R., et al. 2000. Frequency analysis of autoreactive T-helper 1 and 2 cells in bullous pemphigoid and pemphigus vulgaris by enzyme-linked immunospot assay. Br. J. Dermatol. 143:1279-1282.

18. Veldman, C., et al. 2003. Dichotomy of autoreactive Th 1 and Th 2 cell responses to desmoglein 3 in patients with pemphigus vulgaris (PV) and healthy carriers of PV-associated HLA class II alleles. J. Immunol. 170:635-642.

19. Bhol, K., et al. 1995. Correlation of peptide specificity and IgG subclass with pathogenic and nonpathogenic autoantibodies in pemphigus vulgaris: a model for autoimmunity. Proc. Natl. Acad. Sci.U.S. A. 92:5239-5243.

20. Spaeth, S., et al. 2001. IgG, IgA and IgE autoantibodies against the ectodomain of desmoglein 3 in active pemphigus vulgaris. Br. J. Dermatol. 144:1183-1188.

21. Sinha, A.A., et al. 1988. A newly characterized HLA DQ beta allele associated with pemphigus vulgaris. Science. 239:1026-1029.

22. Ahmed, A.R., et al. 1990. Major histocompatibility complex haplotype studies in Ashkenazi Jewish patients with pemphigus vulgaris. Proc. Natl. Acad. Sci.U. S. A. 87:7658-7662.

23. Ahmed, A.R., et al. 1991. Major histocompatibility complex haplotypes and class II genes in non-Jewish patients with pemphigus vulgaris. Proc. Natl. Acad. Sci. U. S. A. 88:5056-5060.

24. Riechers, R., Grötzinger, J., and Hertl, M. 1999. HLA class II restriction of autoreactive $T$ cell responses in pemphigus vulgaris: review of the literature and potential applications for the development of a specific immunotherapy. Autoimmunity. 30:183-196.

25. Hertl, M., Karr, R.W., Amagai, M., and Katz, S.I. 1998. Heterogeneous MHC II restriction pattern of autoreactive desmoglein 3 specific $\mathrm{T}$ cell responses in pemphigus vulgaris patients and normals. J. Invest. Dermatol. 110:388-392.

26. Veldman, C.M., et al. 2004. T cell recognition of desmoglein 3 peptides in patients with pemphigus vulgaris and healthy individuals. J. Immunol. 172:3883-3892.

27. Hacker-Foegen, M.K., Fairley, J.A., and Lin, M.S. 2003. T cell receptor gene usage in desmoglein-3specific $T$ lymphocytes from patients with pemphigus vulgaris. J. Invest. Dermatol. 121:1365-1372.

28. Nishifuji, K., Amagai, M., Kuwana, M., Iwasaki, T., and Nishikawa, T. 2000. Detection of antigen-specific $B$ cells in patients with pemphigus vulgaris by enzyme-linked immunospot assay: requirement of $\mathrm{T}$ cell collaboration for autoantibody production. J. Invest. Dermatol. 114:88-94.

29. Lin, M.S., et al. 2000. Desmoglein-1-specific T lymphocytes from patients with endemic pemphigus foliaceus (fogo selvagem). J. Clin. Invest. 105:207-213.

30. Gebhard, K.L., et al. 2005. Ex vivo analysis of desmoglein 1-responsive T-helper (Th) 1 and Th2 cells in patients with pemphigus foliaceus and healthy individuals. Exp. Dermatol. 14:586-592.

31. Hoffman, M.A., Qiao, X., and Anhalt, G.J. 2003. CD8+ T lymphocytes in bronchiolitis obliterans, paraneoplastic pemphigus, and solitary Castle- 
man's disease. N. Engl. J. Med. 349:407-408.

32. Liu, Z., et al. 1993. A passive transfer model of the organ-specific autoimmune disease, bullous pemphigoid, using antibodies generated against the hemidesmosomal antigen, BP180. J. Clin. Invest. 92:2480-2488.

33. Sitaru, C., et al. 2002. Autoantibodies to bullous pemphigoid antigen 180 induce dermal-epidermal separation in cryosections of human skin. J. Invest. Dermatol. 118:664-671.

34. Büdinger, L., et al. 1998. Identification and characterization of autoreactive $\mathrm{T}$ cell responses to bullous pemphigoid antigen 2 in patients and healthy controls. J. Clin. Invest. 102:2082-2089.

35. Lin, M.S., et al. 2000. Epitopes targeted by bullous pemphigoid $T$ lymphocytes and autoantibodies map to the same sites on the bullous pemphigoid 180 ectodomain. J. Invest. Dermatol. 115:955-961.

36. Hofmann, S., et al. 2002. Severity and phenotype of bullous pemphigoid relate to autoantibody profile against the $\mathrm{NH} 2$ - and $\mathrm{COOH}$-terminal regions of the BP180 ectodomain. J. Invest. Dermatol. 119:1065-1073.

37. Thoma-Uszynski, S., et al. 2004. BP230- and BP180specific auto-antibodies in bullous pemphigoid. J. Invest. Dermatol. 122:1413-1422.

38. Thoma-Uszynski, S., et al. 2006. Autoreactive T and $\mathrm{B}$ cells from bullous pemphigoid (BP) patients recognized epitopes clustered in distinct regions of BP180 and BP230. J. Immunol. 176:2015-2023.

39. Lin, M.S., Gharia, M.A., Swartz, S.J., Diaz, L.A., and Giudice, G.J. 1999. Identification and characterization of epitopes recognized by $\mathrm{T}$ lymphocytes and autoantibodies from patients with herpes gestationis. J. Immunol. 162:4991-4997.

40. Hacker-Foegen, M.K., Zillikens, D., Giudice, G.J., and Lin, M.S. 2004. T cell receptor gene usage of
BP180-specific T lymphocytes from patients with bullous pemphigoid and pemphigoid gestationis. Clin. Immunol. 113:179-186.

41. Black, A.P., et al. 2004. Rapid effector function of circulating NC16A-specific T cells in individuals with mucous membrane pemphigoid. Br. J. Dermatol. 151:1160-1164.

42. Lin, M.S., et al. 2002. Autoimmune responses in patients with linear IgA bullous dermatosis: both autoantibodies and $\mathrm{T}$ lymphocytes recognize the NC16A domain of the BP180 molecule. Clin. Immunol. 102:310-319.

43. Delgado, J.C., et al. 1996. A common major histocompatibility complex class II allele HLA-DQB1* 0301 is present in clinical variants of pemphigoid. Proc. Natl. Acad. Sci. U. S. A. 93:8569-8571.

44. Banfield, C.C., et al. 1998. The association of HLADQ7 with bullous pemphigoid is restricted to men. Br. J. Dermatol. 138:1085-1090.

45. Haase, C., et al. 1998. Detection of IgG autoantibodies in the sera of patients with bullous and gestational pemphigoid: ELISA studies utilizing a baculovirus-encoded form of bullous pemphigoid antigen 2. J. Invest. Dermatol. 110:282-286.

46. Setterfield, J., et al. 2001. Mucous membrane pemphigoid: HLA-DQB1*0301 is associated with all clinical sites of involvement and may be linked to antibasement membrane IgG production. Br. J. Dermatol. 145:406-414.

47. Koch, P.J., et al. 1997. Targeted disruption of the pemphigus vulgaris antigen (desmoglein 3 ) gene in mice causes loss of keratinocyte cell adhesion with a phenotype similar to pemphigus vulgaris. J. Cell Biol. 137:1091-1102.

48. Amagai, M., et al. 2000. Use of autoantigen-knockout mice in developing an active autoimmune disease model for pemphigus. J. Clin. Invest. 105:625-631.
49. Tsunoda, K., et al. 2002. Pathogenic autoantibody production requires loss of tolerance against desmoglein 3 in both T and B cells in experimental pemphigus vulgaris. Eur. J. Immunol. 32:627-633.

50. Aoki-Ota, M., et al. 2004. A mouse model of pemphigus vulgaris by adoptive transfer of naive splenocytes from desmoglein 3 knockout mice. Br. J. Dermatol. 151:346-354.

51. Eming, R., et al. 2005. Ex vivo analysis of T cell epitopes of desmoglein 3 in a HLA class II-transgenic mouse model of pemphigus vulgaris. J. Invest. Dermatol. 125:A12.

52. Sonderstrup, G., et al. 1999. HLA class II transgenic mice: models of the human CD4+ T-cell immune response. Immunol. Rev. 172:335-343.

53. Sakaguchi, S. 2005. Naturally arising Foxp3expressing CD25+CD4+ regulatory $\mathrm{T}$ cells in immunological tolerance to self and non-self. Nat. Immunol. 6:345-352.

54. Veldman, C., Hohne, A., Dieckmann, D., Schuler, G., and Hertl, M. 2004. Type I regulatory T cells specific for desmoglein 3 are more frequently detected in healthy individuals than in patients with pemphigus vulgaris. J. Immunol. 172:6468-6475.

55. Sinha, E., et al. 2005. Elevation of antigen specific CD8+ CD28-cells in newly remittent patients with pemphigus vulgaris. J. Invest. Dermatol. 125:1084A.

56. Veldman, C., et al. 2006. Inhibition of the transcription factor Foxp3 converts desmoglein 3-specific type 1 regulatory $\mathrm{T}$ cells into autoreactive $\mathrm{T}$ helper 2-like cells. J. Immunol. 176:3215-3222.

57. Veldman, C., Nagel, A., and Hertl, M. 2006. Type 1 regulatory $\mathrm{T}$ cells in autoimmunity and inflammatory diseases. Int. Arch. Allergy Immunol. In press.

58. Yancey, K.B. 2005. The pathophysiology of autoimmune blistering diseases. J. Clin. Invest. 115:825-828. doi:10.1172/JCI200524855. 